\title{
PROCEEDINGS
}

OF THE

\section{NATIONAL ACADEMY OF SCIENCES}

Volume 21

February, 15, 1935

Number 2

\section{INERT REGIONS OF CHROMOSOMES AS THE TEMPORARY PRODUCTS OF INDIVIDUAL GENES}

\author{
By H. J. Muller and S. M. Gershenson \\ Institute of Genetics, Academy of Sciences, Moscow \\ Communicated January 8, 1935
}

Soon after the phenomenon of no crossing-over in the male was discovered, it was pointed out (Muller 1914, 1918), that this circumstance must lead to the accumulation of recessive mutations, and even losses of some genes and duplications of others, in any chromosome which, like the $Y$, is constantly carried in heterozygous condition in a sex in which no crossing-over occurs. The lack of active genes in the $Y$ was thus interpreted. It was then taken for granted, however, that the visible material of the $Y$ chromosome was of a nature fundamentally similar to that composing the bulk of other chromosomes, and consisted of more or less inactivated genes together with the usual amount of accessory chromatin and other substances that may normally accompany genes in the chromosome as seen at metaphase. When later it was shown (Muller and Painter, 1932), that the $X$ chromosome had a similarly inert region, homologous to that of a part of the $Y$, it was concluded that the former had probably been derived from the latter by a kind of translocation, and it was naturally assumed that the inert region of the $X$, like that of the $Y$, consisted of inactivated or "degenerated" chromatin.

At this later date, it was, to be sure, realized that the chromosome material of both the $Y$ and $X I$ (the inert region of the $X$ ), might really be relatively devoid of genes, rather than full of degenerated genes, and that the actual gene-string in the inert regions might therefore be much smaller, in proportion to the bulk of visible chromatin, than it was in the "active" regions of the chromatin. But it was found by Oliver (1932) that, in the case of inversions, breaks probably occur in the inert region, as compared with the active region of the $X$, in somewhat the same ratio as that which the bulk of the inert region as seen at metaphase bears to the bulk of the active region (further evidence of this sort will be referred to below), and this was taken by Muller and Oliver as an indication that the inert and active regions were probably constituted in a similar way, an inert region 
thus containing a chromonema of similar length, degree of coiling and breakability to that in an equal amount of an active region (see Oliver, Ibid.). The alternative to this would have been the assumption, which at that time was less plausible, that, length for length of chromonema, breakage occurred more readily in the inactive region, in which the chromonema was shorter (i.e., coiled to a lesser degree).

A review of the evidence now at hand from various sources, however, makes the conclusion now much more probable that the inert regions of the $X$ and $Y$ are fundamentally different in structure from the active regions and do not consist of a row of degenerated genes arranged single file in a coiled chromonema like the functional genes in the active region, but that they consist essentially of non-genic material derived from a very few specific active genes, between which breakage takes place much more readily than between genes in the chromonema of the so-called active region. Whether these chromatin-producing genes of the "inert region" lie in the form of a coiled chromonema in their region of the chromosome at the time of mitosis remains a question that it is still difficult to decide.

Evidence for the above conclusion is to be found in several different directions. Most telling is the fact that in various cases of breakage within the inert region of the $X$ or $Y$ chromosomes, certain large sections of specific size seem to be broken off or carried over en bloc. Thus in all of Stern's and of Kaufmann's cases of exchange between the $X$ and the $Y$, when an arm of the $Y$ was broken off or transferred either the whole of the arm or just half of the long arm was concerned. Again, in the deletions of the $X$ chromosome examined by Painter and Muller (1929; also Muller and Painter, 1929) the size of the piece of the $X$ remaining was sensibly the same in the different cases in which the locus of bobbed was presentamounting to approximately one-third the length of the chromosome, as we would expect if exactly all of the inert region were present, in addition to a negligible section of active region from the left end. In another case (deletion 24, described by Muller and Painter, 1932) in which the bobbed locus was not present, the fragment was much smaller, being only a few times the size of the fourth chromosome. This fragment must have consisted mostly of inert material, since only that part of the active region to the left of scute was present here, and this inert material must be that which is associated with the fibre attachment locus or other unrecognized loci close to the latter. Conversely, in the case of bobbed-deficiency studied by Dobzhansky (1932, exhibited to the Sixth International Congress of Genetics), the deleted chromosome lacked almost exactly that amount of material which was present in the deleted $X$ chromosomes of Painter and Muller, namely, the inactive material, and it contained the amount of material which was absent in the previously described deletions, namely, the active material. This then constitutes evidence from the reverse di- 
rection for the same conclusion, namely, that the inert material is in definite blocks of comparatively large size. The chromosome described by Gershenson (1933), in which by crossing-over between two different inverted chromosomes (scute ${ }^{4}$ and scute $^{8}$, respectively) a deficiency for the region of bobbed was produced, likewise lacks a region nearly equal to the length of the inert region itself, i.e., Gershenson's crossover chromosome and Dobzhansky's deficient chromosome seem to be of sensibly the same length. All this would lead us to the conclusion that, where the bobbed locus was present, nearly all of the inert region was also present, with the exception of a small section-perhaps one-fourth of the total-which is associated with the spindle fibre locus. In the $Y$ chromosome the bobbed region is apparently of similar length to that in the $X$, constituting about one-half of the long arm.

Studies of Panshin and Muller (unpublished) on translocations between the $Y$ chromosome and the fourth, furnish another, though less direct, line of evidence in the same direction. They find that in the great majority of translocations of this type-at least 90 per cent-one or more of the genes of the $Y$ chromosome necessary for the fertility of the male, have become so affected by their altered position as to be no longer capable of producing the effects necessary for fertility. This indicates that these fertility genes are in all these cases located near the point of breakage. Since, however, the $Y$ chromosome at metaphase appears rather long, and since there are probably not many fertility genes, the result is hard to understand, unless we suppose that in the ordinary "resting" stages of functioning cells, these genes lie close together and are not separated by the great bulk of inert material seen at metaphase.

In view of this interpretation, the observation of Painter (1933, 1934a and $\mathrm{b}$ ) that the inert regions of the $X$ chromosome are hardly, if at all, to be found in the chromosome structure of the cells in the salivary glands, becomes understandable, and in fact constitutes further evidence for our interpretation itself. We may suppose that in these stages when the genes are functioning in cell metabolism, they do not produce the great local accretions of material to be observed at metaphase in the inert regions, and that the salivary gland chromosomes, therefore, give a truer picture of the chromonema, in so far as the latter represents a string of real genes in which the different genes occupy comparable lengths of the thread. Whether or not the inert material that was present at metaphase still exists somehow in the resting nucleus, and, if so, what its functions may be, is another question. Its functions in segregation and crossingover likewise remain important subjects for study.

While the above conception disagrees with the idea of the inert region as representing a degenerated gene string, it does not contradict the notion that a process of degeneration and of loss did occur in the past evolution 
of the $Y$ chromosome, owing to the protection from natural selection af forded by its continually heterozygous condition. The loss, however, has probably, by this time, become complete with regard to all genes that are no longer needed, and the material that is seen, instead of consisting of "degenerated" genes and the material accessory to them, existing in the proportions usual for genes and their accessory material, probably consists almost entirely of the accessory material-i.e., of some sort of especially bulky reaction-product-of the few functional genes that still remain. As for the existence of translocations, to account for the continued similarity between the $Y$ and the inert region of the $X$, the reality of the occurrence of such translocations (including "crossovers"), even today, is attested to by findings of Stern and of Kaufmann, previously alluded to.

Attention may at this point be recalled to the fact that, on the view here presented, the finding of Oliver of the occurrence of more inversions affecting the right-hand portion of the $X$ than the left-hand portion, is to be interpreted on the basis that there is a much greater likelihood of breakage between two genes which are connected with the inert region than between any two successive genes of the active region. There are several other series of findings which, on our present conception of the inert region, would lead to the same conclusion. One is the fact that $\mathrm{x}$-rays produce translocations of the $Y$ chromosome with approximately the same frequency as those of the $X$ (Muller and Altenburg, 1930), despite the great dearth of active genes in the $Y$ as compared to the $X$. Secondly, there is the fact (Painter and Muller, 1929, confirmed by later work) that the majority of deleted $X$ chromosomes obtained by crossing irradiated males to yellow females with attached $X$ 's have their right-hand break to the right of carnation (and hence probably in the inactive region) despite the fact that there are many more genes to the left of carnation, which are nevertheless so situated that breaks between them would result in deleted $X$ 's having a high viability in this cross. Thirdly, of a series of ten gross (i.e., not minute) inversions studied by the present authors, which involved position effects in specific loci near the left end of the $X$ chromosome (yellow, scute, white), six have been found to have their right-hand break in the inert region, and only four to have it in any other part of the chomosome. Fourthly, translocations involving the $X$ have their break in the inert region in a large proportion of cases (Patterson, Stone, Bedichek and Suche, 1934).

The excess of breaks in the inert region is in part due to the fact that breaks are more apt to occur near the ends and near the attachment points of chromosomes, as the latter authors show, but this can be by no means the entire explanation. Thus, in work of Levit and of Muller (unpublished), in which the scute 8 chromosome, which has bobbed and most of the inert region located near but not at the left end (and far from the attachment 
point), was irradiated, it was found that most of the deleted $X$ 's formed had their left-hand breaks in the inert region, rather than in the active region to the left of the latter and still nearer the end, although there are probably many more genes in this left terminal active region than in the inert region. Apparently "simple breaks" of the scute 8 chromosome also occur preferentially in the inert region, as shown by the same authors and also by Patterson (1933). The latter breaks occur about five times as often beyond bobbed as between carnation and bobbed, but among deletions and inversions of $X$ 's that were normal before irradiation there seem to be considerably fewer breaks beyond bobbed (between bobbed and the fibre attachment) than between carnation and bobbed, even though the latter interval is further from the end of the chromosome. The breakability, therefore, is in part a specific property of the region concerned, and it is extremely high between genes that lie in the inert region.

On the new conception of the inert region here proposed, various experimental facts require a different interpretation than they would otherwise receive. One example may suffice here. Four different apparently "simple breaks" were produced by Muller and Koerner (unpublished) in the scute 8 chromosome, in the interval between the gene achaete, in the lefthand active region, and bobbed, the left-hand fragment being lost. On the view that the inert region, like the active region, consists of a large number of small, genetically distinct segments (corresponding to genes), the different "truncated" scute 8 chromosomes thus resulting should be of different lengths, lacking not only the left-hand active region (from the left terminus to achaete, inclusive), but also a part of the inert region lying beyond bobbed. They were accordingly tested with the object of determining whether the latter loss were capable of producing any detectable phaenotypic effect. A really adequate test of this kind had not hitherto been possible, since hitherto only individuals deficient for the region including bobbed had been tested, and these necessarily required a $Y$ chromosome to cover their bobbed-deficiency; this $Y$ then might at the same time have covered the deficiency of other genes of the inert region. In the present case crosses were carried out, the genetic details of which it will not be necessary to describe, to allow the production of zygotes homozygous for the truncated $X$ chromosome, but not containing a $Y$ chromosome, and having their deficiency of the active region at the left end compensated for by the presence of a chromosome fragment (the left part of the $X$ of "mottled 5") containing those genes but not containing any part of the inert region of the $X$ (since this fragment of the $X$ is here attached to the right portion of chromosome IV). The results showed clearly that in all cases such females were viable, and the conclusion seemed to follow that the loss of none of the genes in the inert region that normally lay between bobbed and the fibre attachment caused any detectable phaenotypic effect, 
even in the absence of $Y$ : Now, however, we see that the result probably means only that in all these cases the break was at, or very nearly at, the same point-between bobbed and achaete - and that there was no part of the inert region absent. That part which may be connected with the bobbed locus is necessary for life, in so far as the bobbed gene itself is necessary for life. Whether the genes of the inert region that affect variegation and fertility and possibly other characters, are at other loci, and also have a part of the inert region connected with them, is as yet not definitely known, although the evidence above would indicate that most of the in. ert region depends on very few genes. Neither do we know whether the fibre-attachment gene of the $X$ is in any way specific, or may be completely replaced by a fibre-attachment gene from another chromosome.

As most of the evidence for our present interpretation of the inert region depends upon the small series of cases which were observed some time ago without the present question being in view, and as such comparison is subject to considerable error in the estimate of the length of the pieces of chromosome involved, Gershenson is now undertaking a special study and comparison of a more extended series of cases of this sort. This should give a definitive answer to the question of whether or not the inert region is composed of blocks, and of the number and size of such blocks, and the genes with which they are associated.

Gershenson, S., "Studies on the Genetically Inert Region of the X-Chromosome of Drosophila. I. Behavior of an X-Chromosome Deficient for a Part of Its Inert Region," Jour. Genetics, 28, 297-313 (1933).

Kaufmann, B. P., "Interchange between X- and Y-Chromosomes in Attached $X$ Females of Drosophila melanogaster," Proc. Nat. Acad. Sci., 19, 830-838 (1933).

Muller, H. J., "A Gene for the Fourth Chromosome of Drosophila," J. Exp. Zoöl., 17, 325-336 (1914).

Muller, H. J., "Genetic Variability, Twin Hybrids and Constant Hybrids, in a Case of Balanced Lethal Factors," Genetics, 3, 422-499 (1918).

Muller, H. J., and Altenburg, E., "The Frequency of Translocations Produced by Xrays in Drosophila," Genetics, 15, 283-311 (1930).

Muller, H. J., and Painter, T. S., "The Cytological Expression of Changes in Gene Alignment Produced by X-rays in Drosophila," Amer. Nat., 63, 193-200 (1929).

Muller, H. J., and Painter, T. S., "The Differentiation of the Sex Chromosomes of Drosophila into Genetically Active and Inert Regions," Zeit. ind. Abst. Vererb., 62, 316365 (1932).

Oliver, C. P., "An Analysis of the Effect of Varying the Duration of X-ray Treatment upon the Frequency of Mutations," Zeit. ind. Abst. Vererb., 61, 447-488 (1932).

Painter, T. S., "A New Method for the Study of Chromosome Rearrangements and the Plotting of Chromosome Maps," Science, 78, 585-586 (1933).

Painter, T. S., "A New Method for the Study of Chromosome Aberrations and the Plotting of Chromosome Maps in Drosophila melanogaster," Gentics, 19, 175-188 (1934a).

Painter, T. S., "The Morphology of the X-Chromosome in Salivary Glands of Drosophila melanogaster and a New Type of Chromosome Map for This Element," Genetics, $19,448-469$ (1934b). 
Painter, T. S., and Muller, H. J., "Parallel Cytology and Genetics of Induced Translocations and Deletions in Drosophila," Jour. Hered., 20, 287-298 (1929).

Patterson, J. T., "The Mechanism of Mosaic Formation in Drosophila," Genetics, 18, 32-52 (1933).

Patterson, J. T., Stone, W., Bedichek, S., and Suche, M., "The Production of Translocations in Drosophila," Amer. Nat., 68, 359-369 (1934).

Stern, C., "Untersuchungen über Aberrationen des Y-chromosoms von Drosophila melanogaster," Zeit. ind. Abst. Vererb., 51, 253-353 (1929).

\title{
OBSERVATIONS ON THE STRUCTURE OF LIVING SALIVARY GLAND CHROMOSOMES IN SCIARA
}

\author{
By W. L. Doyle and C. W. Metz
}

Department of Embryology, Carnegie Institution of Washington and ZÖOlOgical Laboratory OF The Johns Hopkins University

\section{Communicated January 15, 1935}

Recent investigations on the giant chromosomes of dipteran salivary gland cells by Heitz and Bauer, Painter, Koltzoff and others ${ }^{1}$ have focused attention on several problems for the study of which these chromosomes are particularly favorable. One problem has to do with the internal organization of the chromosomes in relation to genes. Koltzoff and Bridges have both interpreted the structures visible in fixed (Koltzoff also in living) material from this point of view. ${ }^{2}$

Owing to the unusual size and nature of the chromosomes under consideration it seems particularly desirable to determine the nature of their organization in the living condition, and to ascertain how accurately the fixation images reflect this organization. The present paper is devoted to this topic. It is based on a study of conditions in the fungus flies (Sciara) which are unusually favorable for the purpose.

The living cells, in the intact, uninjured gland, have been examined in body fluid, mineral oil, isotonic Ringer's solution and in various hypo- and hypertonic solutions, and have been observed under the microscope at high magnification during the process of fixation in various ways. The following account summarizes the findings. A more extended treatment will be published elsewhere.

Gross Features.-In normal living glands mounted in body fluid surrounded by mineral oil, the nuclei are spherical and bear a fairly constant size relation to the cells containing them. In mammalian Ringer's solution both cells and nuclei swell, indicating hypotonicity of the solution. In hypertonic salt solutions cells and nuclei immediately shrink. In isotonic salt solution they remain, for a short time, essentially unchanged, and appear as they do in body fluid surrounded by mineral oil. 